\title{
Biosynthesis of mycobacterial methylmannose polysaccharides requires a unique 1-0- methyltransferase specific for 3-0-methylated mannosides
}

\author{
Jorge Ripoll-Rozada ${ }^{1,2}$, Mafalda Costa ${ }^{3}$, José A. Manso ${ }^{1,2}$, Ana Maranha ${ }^{3}$, Vanessa Miranda ${ }^{4}$, André Sequeira ${ }^{4}$, M. $^{2}$ \\ Rita Ventura ${ }^{4}$, Sandra Macedo-Ribeiro ${ }^{1,2}$, Nuno Empadinhas ${ }^{3,5}$, Pedro José Barbosa Pereira ${ }^{1,2}$
}

\begin{abstract}
${ }^{1}$ IBMC - Instituto de Biologia Molecular e Celular, Universidade do Porto, 4200-135 Porto, Portugal. ${ }^{2}$ Instituto de Investigação e Inovação em Saúde, Universidade do Porto, 4200-135 Porto, Portugal. ${ }^{3}$ CNC-Center for Neuroscience and Cell Biology, $3004-504$ Coimbra, Portugal. ${ }^{4}$ ITQB - Instituto de Tecnologia Química Biológica, Universidade Nova de Lisboa, 2780-157 Oeiras, Portugal. ${ }^{5}$ IIIUC - Interdisciplinary Research Institute, University of Coimbra, 3004-504 Coimbra, Portugal.
\end{abstract}

jorge.rozada@ibmc.up.pt

Mycobacteria are priority pathogens in terms of drug resistance worldwide and efforts aimed at deciphering their unique metabolic pathways and unveiling new targets for innovative drugs should be intensified. In particular, nontuberculous mycobacteria (NTM) are environmental organisms increasingly associated to opportunistic infections [1] and known to produce methylmannose polysaccharides (MMP). MMP have been implicated in the metabolism of precursors of cell envelope lipids crucial for stress resistance and pathogenesis. Although the functions of MMP remain to be confirmed experimentally, their tight interactions with fatty acids are intrinsically associated to unique and extensive methylation patterns, resulting from the action of hitherto uncharacterized methyltransferases.

In this work, we identified and characterized biochemically a novel mycobacterial methyltransferase (MeT1) that specifically blocks the non-reducing end of a MMP precursor. We crystallized and determined the first X-ray structure of the SAM-dependent MeT1 from M. hassiacum in complex with magnesium and its exhausted cofactor, SAH. In particular, the three high-resolution 3D structures (in space groups $\mathrm{P} 3{ }_{2} 21$ and $\mathrm{C} 222_{1}$; PDB entries $6 \mathrm{H} 40,6 \mathrm{G} 7 \mathrm{D}$ and 6G80) in combination with SAXS data (SASBDB entry SASDDJ6) unveiled a dimeric arrangement of the enzyme in solution and a highly flexible lid important for its catalytic cycle. This structural information, together with molecular docking simulations, allowed the elucidation of the enzyme's reaction mechanism, furthering our knowledge of MMP biosynthesis and providing important tools to dissect the role of MMP in NTM physiology and resilience [2].

[1] Falkinham III, J. O., (2015). Clin. Chest. Med. 36, 35.

[2] Ripoll-Rozada, J., Costa, M., Manso, J. A., Maranha, A., Miranda, V., Sequeira, A., Rita Ventura, M,. Macedo-Ribeiro, S., Pereira, P. J. B., Empadinhas, N. (2019). Proc. Natl. Acad. Sci. U.S.A. 116, 835.

Keywords: Mycobacterium; polymethylated polysaccharides; methyltransferase; S-adenosyl-L-methionine; 3D structure

We thank SOLEIL, ESRF and ALBA for provision of synchrotron radiation facilities, and their staff for help with data collection. This work was funded in part by national funds through Fundação para a Ciência e a Tecnologia (Portugal) through PhD Fellowship SFRH/BD/101191/2014 (to M.C.); the European Social Fund through Programa Operacional Capital Humano in the form of Postdoctoral Fellowship SFRH/BPD/108004/2015 (to J.R.-R.); the European Regional Development Fund (FEDER), through Centro2020 Project CENTRO-01-0145- FEDER-000012-HealthyAging2020 in the form of a postdoctoral fellowship (to A.M.); and the COMPETE 2020-Operational Programme for Competitiveness and Internationalization (POCI), PORTUGAL 2020 in the form of projects POCI-01-0145-FEDER-029221 (PTDC/BTM-TEC/29221/2017), "Institute for Research and Innovation in Health Sciences", (POCI-01-0145-FEDER-007274), UID/NEU/04539/2013, and Research Unit MOSTMICRO (UID/CQB/04612/2013). 